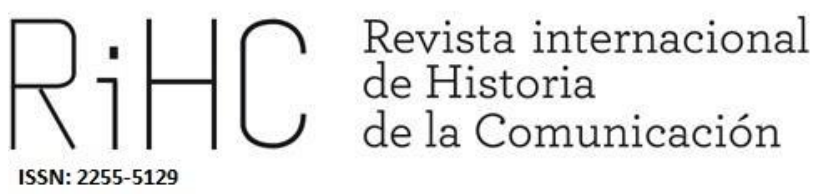

\title{
LA ERA DEL RADIUM: RADIACTIVIDAD Y PUBLICIDAD DE PRODUCTOS MILAGRO EN LOS ALBORES DEL SIGLO XX EN ESPAÑA
}

The radium Era: radioactivity and advertising of miracle products in the dawn of the 20th century in Spain

DOI: https://dx.doi.org/10.12795/RiHC.2020.i14.04

Recibido: $16 / 03 / 2020$

Aceptado: 22/05/2020

Publicado: $15 / 06 / 2020$

Enrique Bordería Ortiz

ORCID (iD) 0000-0002-9388-1139

Universitat de València, España

enrique.borderia@uv.es 
Resumen: El descubrimiento de la radiactividad a finales del siglo XIX proporcionó el conocimiento de un nuevo fenómeno natural que fue utilizado como reclamo sin validación científica en un repertorio de productos, remedios y terapias que en el primer cuarto del siglo XX se anunciaron profusamente en la prensa española. Las experiencias de los manantiales con el agua radiactiva o los emanatorios urbanos fueron algunos de los casos más frecuentes de esas terapias o productos milagro en los que la publicidad de la época utilizaba la ciencia desde el abuso, el desconocimiento o las falsas atribuciones para prometer curaciones para todo tipo de dolencias sin ninguna efectividad práctica.

Palabras clave: Historia de la publicidad, Historia del periodismo, productos milagro, radiactividad, radium.

\begin{abstract}
The discovery of radioactivity at the end of the 19th century provided the knowledge of a new natural phenomenon that was used as a claim, although without any scientific validation, in a repertoire of products, remedies and therapies that in the first quarter of the 20th century were massively announced in the Spanish press. The experiences of the springs with radioactive water or the urban emanating ones were some of the most frequent cases of those therapies or miracle products in which the publicity of the time used science ranging from the abuse to the ignorance or the false attributions in order to promise cures for all type of ailments without any practical effectiveness.
\end{abstract}

Keywords: Advertising history, History of journalism, miracle products, radioactivity, radium.

\title{
Introducción y metodología
}

Hemos realizado un análisis de la presencia de los términos, radioactividad y radium en los contenidos de las publicaciones periódicas españolas desde el 1 de enero de 1900 al 31 de diciembre de 1929 a partir de la Biblioteca Virtual de Prensa Histórica, la Hemeroteca Digital de la Biblioteca Nacional de España, La Hemeroteca Histórica del $A B C$ y la de La Vanguardia. El estudio ha considerado la presencia de esos términos tanto en su cartelera comercial como en sus contenidos periodísticos, con el propósito de conocer primero la difusión de las teorías científicas de la radiactividad, pero sobre todo para exhumar el sorprendente catálogo de anuncios que durante la época emplearon de manera espuria la apelación a la contribución de la radiactividad para curar enfermedades o beneficiar a la salud con la exposición a sus efectos, en un claro ejemplo de lo que fue en ese primer tercio del siglo XX uno de los casos más espectaculares de la llamada publicidad de productos milagro. A partir de más de 25.567 registros, la mayor parte de ellos repeticiones de anuncios comerciales, hemos seleccionado aquellos casos más destacados por la tirada de las publicaciones donde aparecían o por el número de dichos anuncios a lo largo del tiempo, para trazar una aproximación a lo que significó el uso del progreso científico como coartada, en este 
momento en los inicios del conocimiento del fenómeno de la radiactividad, para la publicidad engañosa.

\section{La publicidad sigue a la Ciencia}

En 1898 el matrimonio Curie, Marie y Pierre, presentaba a la comunidad científica un sensacional descubrimiento. Un nuevo mineral, al que bautizaron con el nombre de Radio, que era capaz de emitir unas radiaciones muchas más penetrantes que las desprendidas por otro mineral, el uranio, a través del cual Becquerel en 1896 había descubierto el fenómeno de la radioactividad natural; una propiedad por la que algunas sustancias emitían unos rayos capaces de impresionar una especie de fluorescencia sobre una placa fotográfica, con un poder desconocido.

El nuevo elemento químico, el radio, se descubría como intensamente radiactivo, capaz de atravesar el papel y los tejidos en los experimentos del matrimonio Curie; capaz de emitir lo que describían como una sobrecogedora luz que como un material mitológico parecía venir a alumbrar una nueva era del conocimiento científico. $Y$ así fue, porque a partir de estos momentos se inauguraba un tiempo nuevo que iba a forjar un avance desconocido hasta entonces en el conocimiento de la materia en sus más básicos constituyentes, abriendo el siglo de la radiactividad, susceptible de todo tipo de progresos y avances.

Podemos hablar sin temor a exageraciones del inicio de una Era del Radio, marcada por las prodigiosas características y capacidades que se podían intuir al elemento recién hallado y bautizado. El progreso científico, tras la gigantesca revolución desencadenada por la electricidad a lo largo del siglo XIX disponía de un nuevo aporte con el que continuar la transformación del mundo. No es de extrañar que la prensa se volcara de manera entusiasta en la divulgación del fenómeno de la radiactividad y saludara alborozada el descubrimiento del radio considerándolo el "mayor hallazgo de la historia" (Chicago Daily Tribune, 21/6/1903, citado en Moore, K. 2018: 21).

El radium, como se le denominó en casi todos los países, manifestaba en primer lugar una extraordinaria capacidad de abrirse paso entre los tejidos corroyendo lo que atravesaba. Una suerte de luz mágica y poderosa a la que inmediatamente se le buscaron posibilidades terapéuticas, por ejemplo, en la lucha contra el cáncer destruyendo los tejidos malignos. Los lectores de La Correspondencia de España en los inicios de la centuria podían leer ya una artículo publicado en su portada relatando las investigaciones experimentales hechas en el hospital de Middelsex en Connecticut, EE.UU, donde se decía haber conseguido curar varios casos de Lupus y de úlceras cancerosas. El remate de la noticia era una espita abierta a las más variadas aplicaciones: 
"La acción del radium sobre la sangre actúa como revulsivo y contiene el desarrollo de las invasiones micróbicas" (La Correspondencia de España, 24/1/ 1904).

El aprovechamiento comercial de esas primeras investigaciones fue inmediato, por más que las nuevas terapias apenas estaban en un estado embrionario, y los tratamientos ofertados no podían ser una realidad. La Vanguardia incluye un anuncio en una fecha tan temprana como 1905 en el que un supuesto Dr. Roura Oxandaberro ofrecía ya la curación del cáncer a través de un nuevo tratamiento del radium (Almudéver-Campo, 2007: 207).

La lucha contra el cáncer era sólo la primera y embrionaria utilidad, de un mineral que por el impacto radiactivo se erigió muy pronto en un elemento fabuloso, abierto a enormes potencialidades y ensoñaciones. El razonamiento era muy básico, si el radium podía curar el cáncer, la enfermedad temida, que otras cosas no podría remediar.

La prensa española pronto se empezó a hacer eco de los progresos científicos de la recién descubierta radiactividad con una mezcla de asombro y entusiasmo por las esperanzas que se abrían. Las primeras referencias al radium aparecen a inicios de 1901 en revistas especializadas como Madrid Científico. Y uno de los primeros artículos de la prensa generalista sobre el tema es el que publica La Vanguardia el 19 de junio de 1901 divulgando el hallazgo de Becquerel y los progresos del matrimonio Curie. A partir de 1903 la radiactividad es un asunto que se difunde entre las revistas ilustradas de la época como Blanco y Negro, Nuevo Mundo o La Ilustración Artística, dando fe de la popularidad del asunto.

En general se alababan las supuesta propiedades del radium y su disparatado precio ${ }^{1}$; se relataban las emanaciones de nuevo y misterioso metal, de la sustancia mineral resplandeciente. Primero con los inevitables titubeos, pero pronto fueron deslizándose hacia informaciones donde se conferían a la radiactividad las más diversas capacidades. En esa línea, La Correspondencia de España, uno de los periódicos que más activo se mostró en la difusión de noticias referidas al nuevo elemento, al tiempo que también fue uno de los que más lo incluyó en su cartelera comercial, se refería en diversas noticias a sorprendentes y prodigiosas aplicaciones. El 2 de noviembre 1912 informaba de un supuesto químico japonés, jefe del laboratorio de Tokio, llamado Peyote (sic) que había conseguido cosechas al minuto aplicando el radio a las semillas. El texto era una clase magistral de jerga científica absurda más propia de los futuros seriales de ciencia ficción para los adolescentes: "Aplicando esa energía binaria a una planta en flor, bien pronto los ovarios se convirtieron en frutos fecundos y maduros. Desarrollando en cuatro puntos distintos de un pequeño terreno una corriente catalítica bastaron dos

\footnotetext{
${ }^{1}$ La Correspondencia de Valencia, 14 de enero de 1904, lo utiliza incluso para un chiste: "Un químico habla con un banquero/ -¿Cuánto -dice el 10- da V. en dote a su hija?/ -iCuarenta mil duros!/ -Es muy poco/ ¿Y V. a la suya?/ -Mucho más, dos gramos de Radium.
} 
horas para que simientes se convirtieran en plantas maduras". Podemos imaginar que si las noticias de la época se permitían estas licencias científicas, que no iba a hacer la publicidad con las mágicas oportunidades del radium.

Era una deriva inevitable, como hemos anticipado, que la radioactividad, el radium, acabara siendo utilizado por la publicidad como un reclamo comercial poderosísimo. Tal fue su popularidad en la sociedad del momento que incluso una cupletista y bailarina sevillana, Emilia Montserrat, relativamente famosa entre 1911 y 1923, escogió el nombre artístico de La Radium para su promoción en los escenarios españoles.

En estos albores del siglo XX la prensa española ya había incorporado la publicidad comercial como integrante decisivo de sus páginas y como fórmula segura para aumentar ingresos y multiplicar beneficios. La publicidad había hecho su irrupción a partir de los años 30 del siglo anterior en la prensa española, y un parte sustancial de sus anuncios incluirán todo tipo de productos, remedios y artilugios dedicados a la salud, la belleza y el bienestar en general. Pero como detalla el profesor Laguna $(2017: 35,36)$ es a partir de principios del siglo XX cuando irrumpe la publicidad moderna con profundas cargas persuasivas y sobre todo acompañada por el refuerzo de toda una serie de novedades tecnológicas, como la electricidad, al servicio de la juventud y la belleza; de la salud, y el bienestar, añadiríamos nosotros.

En sus inicios la publicidad de productos milagrosos, que es tan antigua como el oficio de los vendedores ambulantes, charlatanes y embaucadores varios, se asienta sobre la venta de remedios que invocan las más variadas presentaciones, desde los jarabes hasta los ungüentos, pasando por los tónicos, las gotas o las pastillas. Productos que remiten de manera más o menos explícita a sustancias naturales que a partir de plantas, minerales $u$ otras sustancias se venían utilizando ancestralmente; ofrecían una suerte de farmacopea modernizada.

La revolución científica y tecnológica que experimenta su gran salto adelante a finales del siglo XIX e inicios de XX, dota a esa publicidad engañosa de todo un enorme arsenal de nuevas sustancias, nuevos fenómenos naturales, efectos recién descubiertos de valor incalculable para sus discursos de persuasión, engaño y falsas atribuciones de propiedades de los artículos que publicita. La ciencia se encuentra en un espacio de conocimiento basado en el cambio permanente, en el ensanchamiento de sus fronteras a golpe de experimentación, comprobación y verificación de acuerdo con los principios del método científico. La publicidad engañosa de productos milagrosos lo que hace es apropiarse de esas fronteras desdibujadas en las que aún se ha asentado la certeza para divulgar pretendidas bondades y efectos más allá de lo que se haya podido probar. Cuentan con un aliado que es la credibilidad del medio en el que se anuncian tiene para sus lectores. Esos periódicos con sus noticias políticas, sociales o culturales ilustran al público sobre el mundo, relatan los conocimientos científicos y al tiempo presentan 
unos anuncios comerciales que prometen toda una serie de efectos beneficiosos de los productos que tratan de vender a sus lectores.

La ciencia es utilizada por la publicidad, desde esos momentos y hasta hoy en día, como fuente de autoridad por su prestigio como palanca en el cambio del orden material de manera que la calidad de lo anunciado viene refrendada por su aplicación. Se crea un estereotipo de la ciencia como garantía de certeza, común en la prensa escrita (Campanario, 2001: 46).

En nuestro caso además, lo que hemos denominado la publicidad moderna asiste al descubrimiento de la electricidad y el magnetismo primero, o más tarde los Rayos $\mathrm{X}, \mathrm{y}$ aprovecha toda la confusión que despertaba el desconocimiento de los efectos y las aplicaciones de la radiactividad para apelar primero a ese pretendido discurso del conocimiento verdadero y absoluto, para progresivamente ir incorporando otro discurso persuasivo pseudocientífico al servicio de unos productos que prometían las curaciones nunca vistas. Como se dirigían y se dirigen a un público mayoritariamente lego en cuestiones científicas, el embuste era tanto más efectivo.

\section{Productos radioactivos, milagros mortales}

La irrupción de las milagrosas propiedades del radio fue generalizada en el orbe occidental. Kate Moore (2018: 21-22) en el estudio que realiza del caso de un grupo de mujeres norteamericanas que se envenenaron en la época de la I Guerra Mundial empleando el radio, sin ningún tipo de protección, para pintar las esferas de los relojes de pulsera y conseguir que fueran luminiscentes en la oscuridad, detalla la fiebre que sobre la publicidad en el país americano irradió ese nuevo material. Habla de los farmacéuticos que vendían píldoras y vendajes radiactivos; de los anuncios de una jarra con revestimiento de radio que convertía en radiactiva el agua que se metía en ella. $Y$ mucho más: "Se vendían suspensorios y ropa interior con radio, mantequilla con radio, leche con radio, dentífrico con radio (garantizaba una sonrisa más blanca) y hasta un maquillaje y cremas faciales con radio". 


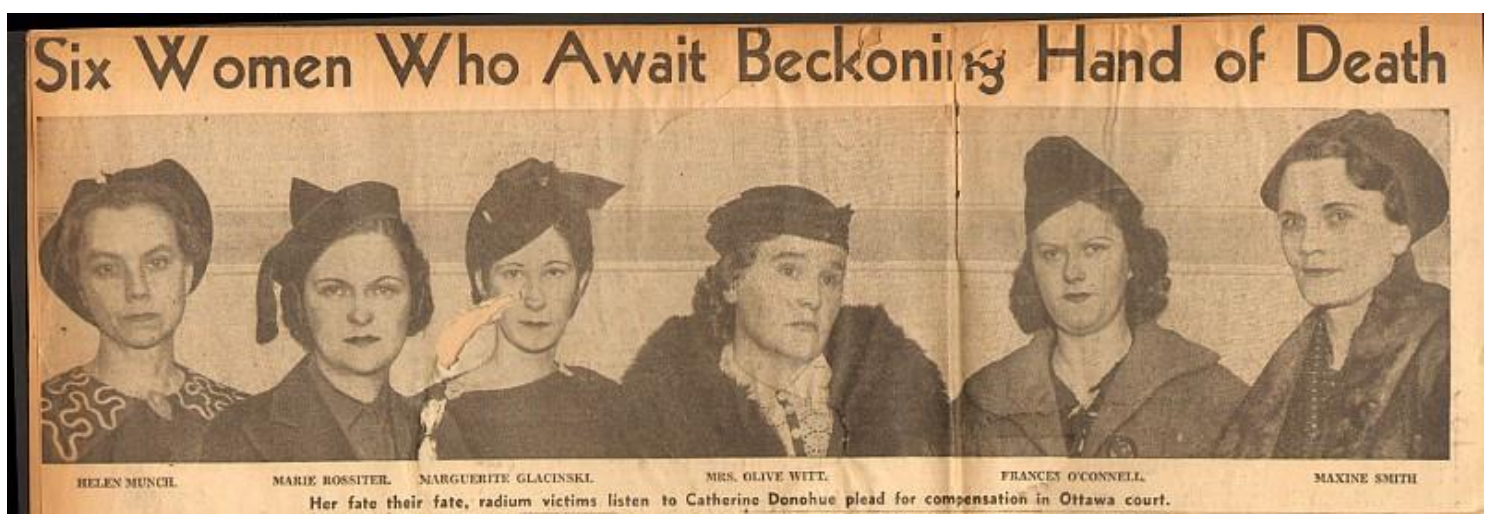

IMAGEN 1. Trabajadoras de la fábrica United States Radium Corporation, conocidad como las "Radium Girls". Daily Times, Chicago's Picture Newspaper, 1938.

En Estados Unidos uno de los casos más espectaculares fue el de llamado Radithor, un agua destilada comercializada en la década de los 20 a la que se incorporaron isótopos de radio Ra226 y Ra228, de las que el empresario William Baley, aunque no poseía ninguna titulación universitaria pese a su paso por Harvard de donde fue expulsado por mala conducta, comercializó más de 400.000 botellas para curar enfermedades mentales, pero también la impotencia masculina. El lema de su publicidad era " $A$ perpetual sunshine" (Dragoni, 2012: 109-110). Por su elevado coste estaba destinado a las clases más pudientes. El escándalo estalló cuando en 1932 se produjo la muerte de Eben MacBurney, un multimillonario habitual de la prensa popular, ocasionada por un envenenamiento por radio ${ }^{2}$, tras la que se descubrió que MacBurney había estado bebiendo un frasco de Radithor al día durante varios años en su afán por impulsar su vigor sexual. Una investigación pública acabó revelando los peligros de la exposición al radio y aunque Baley no fue condenado acabó cerrando sus laboratorios. La prueba de que las autoridades no persiguieron al farsante fue su testimonio de que el cierre de sus negocios radiactivos en los años treinta fue debido más a la falta de éxito debido a la Gran Depresión, no olvidemos que hablamos de productos de precios muy elevados, que a sus continuos problemas con la Comisión Federal de Comercio (https://www.orau.org/ptp/collection/quackcures/radith.htm).

Pero antes, el éxito del Radithor le llevó a comercializar y anunciar otros productos como el Bioray, que era un pisapapeles radiactivo; el Adrenoray, una hebilla radiactiva para el cinturón que reforzaba el vigor sexual masculino, o el Radioencrinator que también alcanzo notable popularidad en EE.UU. Este fue un artefacto producido entre 1922 y 1929, también con isótopos de radio y con forma de tarjeta de crédito que estaba destinado a introducirse en la ropa interior del hombre y usarse debajo del escroto durante un período prolongado. En realidad, el producto que estaba muy lejos de causar

\footnotetext{
${ }^{2}$ El cadáver de MacBurney fue enterrado en un ataúd de plomo y cuando fue exhumando en 1965 para su estudio, el cuerpo aún emanaba altos niveles de radiactividad. (https://www.labrujulaverde.com/2017/02/el-caso-radithor-y-la-moda-de-los-medicamentosradiactivos-de-los-anos-veinte)
} 
un impulso a la potencia masculina; al contrario, usar un Radiendocrinator de acuerdo con las instrucciones del fabricante probablemente habría llevado a una esterilidad temporal y, por supuesto, a un mayor riesgo de cáncer. En otras palabras, era un tipo de anticonceptivo masculino.

En Europa también aparecieron los anuncios de productos que explotaban las bondades del radio. En Alemania se comercializó el chocolate Radium fabricado por Breuk\&Braun y el pan Radium de la panadería Hippman-Blach, hechos ambos con agua de radio hasta que dejaron de venderse en 1936. En Francia fue popular la crema Tho-Radia creada en 1932 por el farmacéutico Alexis Moussalli, aunque en el anuncio se atribuía muy oportunamente su creación a un tal doctor Alfred Curie, que incorporaba en su fabricación tierras raras con torio y radio para acabar con las arrugas de la cara. Llegados a este punto sobra cualquier coletilla sarcástica.

Podemos imaginar la alarma que nos pude despertar hoy en día, cuando conocemos los mortales efectos de la radiación, el empleo alegre de ese elemento en productos de consumo cotidiano. Es cierto que no faltaron casos, como los referidos o el asunto de "Las chicas del radio ${ }^{3 "}$ en los que la imprudencia y falta de protección provocaron no pocas muertes, pero en realidad la presencia del radio en la mayoría de productos no era habitual sino apenas una apelación retórica oportunista. Era muy costoso de obtener ${ }^{4}$ y de un precio elevadísimo. Los propios periódicos españoles se asombraban como hemos apuntado ya en su momento de esa cotización que oscilaba entre los $300.000^{5}$ y $400.000^{6}$ francos el gramo según sus propias afirmaciones. Resultaba, pues, bastante iluso, pensar que ese elemento al que se atribuía ser el más caro, con diferencia, del mundo, pudiera emplearse de manera generalizada y masiva en los más diversos usos.

La singularidad del caso español es que la explotación de la radiactividad como reclamo comercial se concentró en un grupo reducido de remedios, pero que en todo caso fueron ampliamente publicitados durante años. Buena parte de los artilugios y fármacos comercializados en Estados Unidos y Europa no llegaron al mercado español.

Al margen de ellos, de los que hablaremos más extensamente en los siguientes epígrafes, sólo podemos referir la publicidad muy ocasional de un puñado de productos que iban desde la higiene personal a los tradicionales tónicos, ahora modernizados por

\footnotetext{
${ }^{3}$ Decenas de las trabajadoras de la fábrica United States Radium Corporation que afinaban los pinceles impregnados de radio con los que pintaban las esferas de los relojes simplemente chupándolos, murieron en los primeros años de la década de los años 20, víctimas de devastadores osteosarcomas, un tipo de cáncer de hueso. (Moore, 2018)

${ }^{4}$ Los propios Curie en sus investigaciones utilizaron toneladas del mineral de uranio, la pechblenda, para varios años después obtener 0 '1 gramos de sal de radio.

${ }^{5}$ El Radical, 20/2/1910.

${ }^{6}$ La Correspondencia de España, 6/6/1910.
} 
efecto de la radiactividad o los burdos engaños de timadores con cachivaches absurdos. Ninguno de ellos tuvo una especial continuidad.

Ya a inicios de 1906 la prensa anunciaba la loción RADIUM proveniente de un laboratorio francés de París para regenerar el cabello y combatir la calvicie. Prometía que "aplicada sobre el cuero cabelludo detiene en algunos días la caída del cabello, sea cual sea la causa, da a la raíz una savia tal, un vigor tal, que la cabellera vuelve a crecer más hermosa y opulenta que nunca" (El Cantábrico: diario de la mañana, 24/2/1906). No contentos ya con esto, en el anuncio se aseguraba que devolvía el color a los cabellos grises, combatía la caspa, los eczemas, y otras afecciones del cuero cabelludo. Los remedios del producto se vinculaban a la idea fortificadora de la radiación; el radium como fuente de poder.

En la categoría de artículos de mercachifle encontramos El Talismán de la Felicidad, "Una sortija misteriosa que fortalece por radio-actividad odo-electroide el dinamismo humano" y que prometía fortuna, salud y felicidad; suerte, amor y dicha (La Correspondencia de España, 12/6/1909).

En otra línea, el diario tinerfeño La Opinión anunciaba en sus páginas el Toniradium Veldiz: "Este (sic) el mejor tónico y vigorizante conocido á base de compuestos fosforados provistos de radio-actividad, por asociación especial con el Radium y que les hace completamente asimilables" (La Opinión, 5/7/1909).

Y un año después aparecía en prensa un anuncio ilustrado por el dibujo de un gran pabellón auricular rematado con la palabra "Sordos" en un cuerpo de gran tamaño, en el que se prometía la curación de la sordera con el radium y emplazaba al lector para su adquisición a un establecimiento de la calle Montera de Madrid (La Correspondencia de España, 21/9/1910); eso sí debemos admitir que fue un anuncio sin continuidad.

En fechas más tardías, fuera ya del momento álgido del radio en España, encontramos uno de los pocos anuncios internacionales que tuvo presencia en la prensa española. Hablamos del caso singular de la publicidad de la pasta de dientes radiactiva Doramad, producida en Alemania por la compañía Auergesellschaft de Berlín desde la década de 1920 hasta la Segunda Guerra Mundial. El dentífrico, al parecer, contenía una minúscula cantidad de torio y se anunció en varias publicaciones de la España insular como el diario republicano La Prensa a lo largo de 1927, y el diario católico de Menorca El Iris, durante 1932. En su publicidad entre otras propiedades destacaba. "Su acción antiséptica que desarrolla al máximum del elemento radio-activo, destruye radicalmente los microorganismos generales de la caries, piorrea y demás males de la boca" (El Iris, 16/12/1932). Más adelante en 1937 se anunció en el vespertino Falange de Las Palmas de Gran Canaria, creado tras el golpe militar de 1936, donde se extendía y prometía que "La pasta dentífrica Doramad por causa de sus componentes radio-activos facilitaba la salivación, evitando el sarro y la producción de ácidos perjudiciales en la boca" (Falange, 
8/6/1937). Este representa un caso excepcional por la fecha tardía en que se anunciaban las pretendidas bondades y efectos terapéuticos de la radioactividad porque desde finales de los años 20 e inicios de los 30 ya estaban difundiendo los peligros de la exposición a las sustancias radiactivas. En una fecha relativamente temprana, 1923, la prensa española empezaba advertir de esos efectos negativos: "Hay que andarse con sumo cuidado en las aplicaciones del radio. Produce, ciertamente, resultados sorprendentes de curaciones maravillosas; pero también produce, usado indebidamente, terribles efectos de llagas y quemaduras de curación difícil" (EI Imparcial, 11/7/1923).

\section{La Batalla del voltio. Balnearios radiactivos}

El reclamo de la radiactividad se concentró en el caso español en un sector muy específico, el del agua, convertida por acción de la recién descubierta manifestación, en un nuevo producto milagro, al que ampliar los supuestos terapéuticos ya atribuidos al líquido elemento desde tiempo ancestrales. La publicidad tuvo dos escenarios complementarios, los balnearios y el agua embotellada, que en buena parte provenía de esos mismos lugares.

El turismo de balnearios se convierte en un gran negocio durante el siglo XIX e inicios del siglo XX en toda Europa. España no permaneció al margen, aunque con una periodización diferente, del entusiasmo registrado por los balnearios a principios del siglo XX. "Al lado de los balnearios tradicionales, recuperados y en su caso remodelados, con usuarios de extracción popular y ámbito regional, surgieron grandes establecimientos en forma de villas termales. Estas estaban concebidas no sólo como centros de salud sino como lugares de descanso veraniego y de relación social, dirigidos hacia una clientela refinada de alto poder adquisitivo" (Vallejo-Pousada, 2015: 64).

En la mayoría de los establecimientos que hemos analizado nos encontramos con historias muy similares. Muchos de los impulsores o dueños de los balnearios eran emigrantes que regresaban enriquecidos del continente americano, los conocidos como indianos, y que invertían parte de sus fortunas en la modernización y engrandecimiento de tradicionales establecimientos termales ahora dotados de hoteles y residencias más lujosas (Vilar Rodríguez, 2014: 15), como fue el caso de Casimiro Gómez en el balneario de Lérez (Pontevedra), Elisardo Dominguez en el balneario de Acuña (Pontevedra) o José Salmerón en la Aliseda (Jaén). 


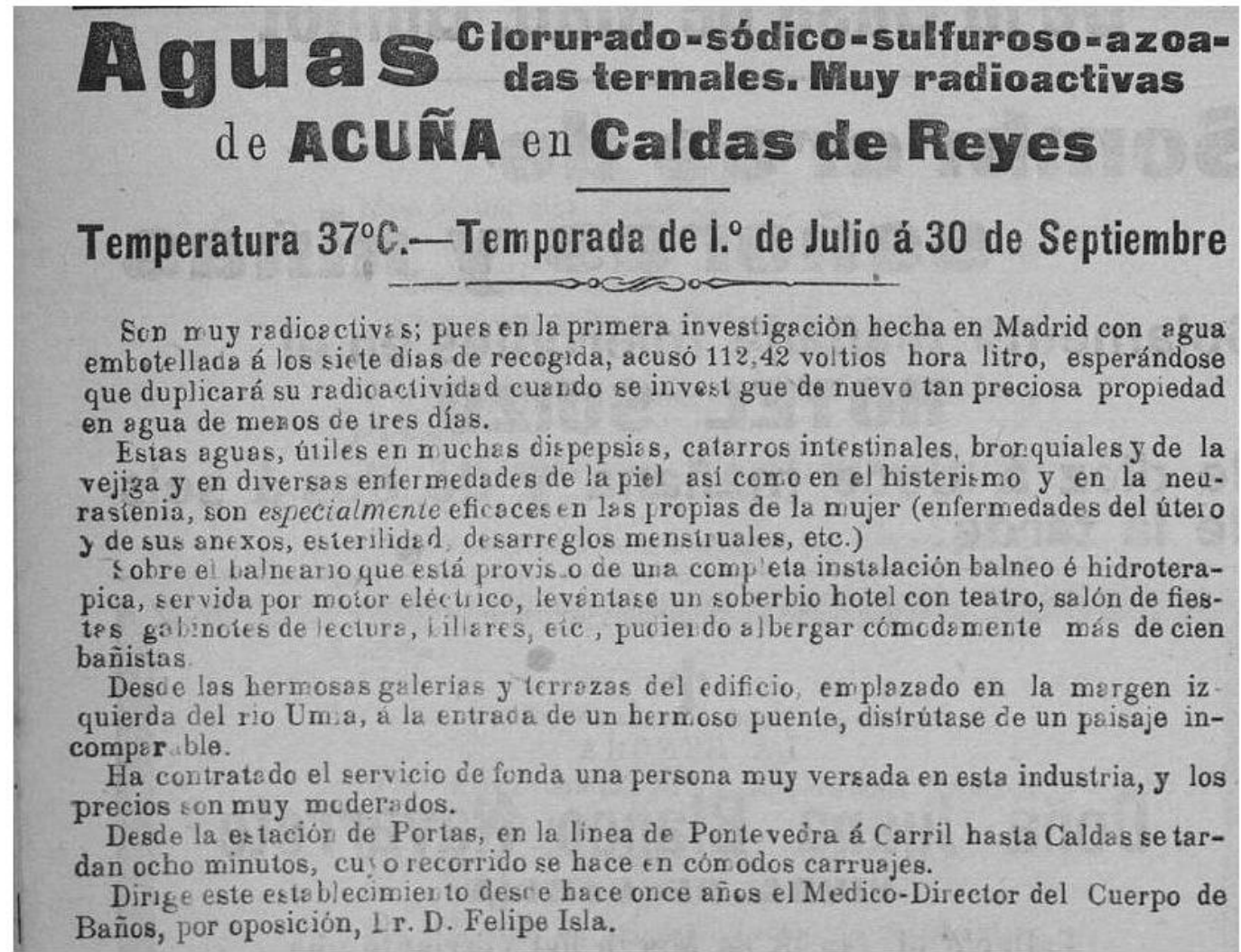

IMAGEN 2. Publicidad del Balneario de Aguas radiactivas de Acuña en Caldas de Reyes en El Eco de Santiago 15 mayo 1906

Es a partir de la primavera de 1906 cuando en los frecuentes anuncios de los balnearios empieza a utilizarse el reclamo de la palabra radiactivo como elemento de diferenciación y valor añadido.

Resulta muy interesante uno de los primeros ejemplos, el Balneario de Cestona en Guipúzcoa, que venía anunciando sus aguas desde hacía décadas en la prensa, pero al calor del nuevo fenómeno, el 6 de abril de 1906 incluían por primera vez el término radiactividad, concretamente señalaba que sus aguas eran "las únicas para el hígado, bazo, estómago, intestinos, etc.; poseen mucha más radiactividad que las aguas de Carlsbad"' (La Correspondencia de España, 6/4/1906). El uso de lo radiactivo se mantuvo en Cestona, al menos hasta 1910, siendo los momentos álgidos de las inserciones publicitarias cuando arrancaban las campañas turísticas, entre abril y julio.

La contestación no se hizo esperar. El 10 de mayo de 1906 un anuncio en El Eco de Santiago de las Aguas de Acuña en Caldas de Reyes, destacaba entre sus propiedades

\footnotetext{
${ }^{7}$ El Balneario de Carlsbad, el actual Karlovy Vari en la República Checa era una ciudad balnearia fundada en 1350 de fama mundial entre cuyos huéspedes más famosos destacaron los nombres de Goethe, Chopin, Beethoven o Pedro El Grande, y por tanto referente y modelo para cualquier otro establecimiento en España.
} 
que eran muy radiactivas e indicadas desde los catarros hasta el histerismo, pasando por las "específicamente propias de la mujer (enfermedad del útero y sus anexos, esterilidad, desarreglos menstruales)". Parecía evidente que la fabulosa radiactividad era una cualidad de la que presumir ante los potenciales clientes, más allá de su veracidad. El agua convertida en la navaja suiza con infinidad de utilidades. Además se encargaba de pregonar el nivel de radioactividad fijado en los 112,42 voltios hora litro ${ }^{8}$, precisando una singularidad que los demás no tardarían en imitar.

De manera repentina España parecía haberse convertido en un gigantesco solar radiactivo, una especie de Chernobyl anticipado, que confería espectaculares posibilidades a los establecimientos termales. No había región o zona que quedara al margen de la fiebre radiactiva. La Voz de Alicante dedicaba el 12 de mayo de 1906 un reportaje a ilustrar las bondades de las históricas aguas termales de Busot (Alicante) y en un momento del texto se deslizaba sus propiedades radiactivas para enfatizar sus cualidades. La publicidad más intensa de su valor radiactivo se desarrolló entre 1911 y 1917, especialmente a través de las páginas de El Diario de Alicante, pero desde planteamientos modestos en las cifras porque los niveles de radiactividad fijados para sus aguas se situaban entre los 37,33 a los 40,55 voltios.

Esos primeros anuncios se convirtieron en el pistoletazo de salida de lo que hemos venido en llamar La Batalla del voltio para escenificar la pugna que se estableció entre los distintos balnearios de la época para presumir de las aguas más radiactivas, exhibiendo una constante escalada de cifras en lucha por acumular mayores propiedades.

En mayo se publicitaban las Aguas Medicinales de Vallfogona de Riucorp en Tarragona, y como no podía prescindir de la moda del momento, anunciaban orgullosos que contenían Radium (Diario de Reus, 26/5/1906), al tiempo que recordaban su balneario moderno y sus chalets de alquiler. Con poca modestia decían que "Curan directamente cualquier enfermedad en general y en particular el artritismo, tumores blancos, herpes, úlceras y obesidad". Aparecía aquí, por primera vez esa palabra mágica, radium, como

\footnotetext{
${ }^{8}$ En esos inicios del estudio del fenómeno radiactivo, las formas de medir su presencia e intensidad poco tenían que ver con las actuales. El contacto de las aguas con minerales radioactivos en su proceso de filtrado entre las rocas puede conferir ciertas propiedades radiactivas a las mismas, en todo caso en magnitudes insignificantes. Los anunciantes de la época jugaban con el desconocimiento general y la predisposición positiva del nuevo fenómeno para magnificar esas propiedades y la expresaban en voltios. Los valores utilizaban estas unidades de medida porque se utilizaban unos aparatos llamados electroscopios que registraban la capacidad ionizante del líquido. El problema era doble, primero, porque la medición con esos aparatos era desigual, inexacta y difícilmente comparable, y además porque no había ninguna certificación de esos valores, más allá de la simple voluntad del dueño del manantial en la mayor parte de casos (https://www.investigacionyciencia.es/blogs/fisica-y-quimica/24/posts/aguas-mineralesradiactivas-11822). Por eso algunas cifras eran elevadísimos, en una clara carrera por ofrecer el agua más radiactiva, supuestamente la más beneficiosa, aunque en realidad ninguna había cambiado con los nuevos descubrimientos científicos y si bien no constituía un método de curación, tampoco representaba una amenaza en caso de ser reales tales niveles.
} 
seña de diferenciación y de valor para esas aguas, aunque el que se popularizó fue el de su efecto, la radiactividad.

Poco después se sumaban las Termas de Arnedillo en La Rioja y en una noticia atravesada por la publicidad se detallaba que según el análisis del Instituto de Radiactividad del Instituto de Ciencias de Madrid ${ }^{9}$ sus aguas presentaban una radiactividad de 1142,28 voltios litros, que era hasta la fecha la cifra más grande para la aguas termales en España y el extranjero (La Rioja, 1/7/1906). Era un golpe en la mesa para proclamarse vencedor en este primer round de la Batalla del voltio. El reclamo se mantendría en su publicidad hasta 1915. Sus anuncios se extendieron por un amplio repertorio de prensa regional: Diario de Burgos, El Noticiero de Soria, El Eco de Navarra, El Avisador Numantino, Heraldo Alavés, El Día de Toledo, etc.

La gran campaña, por sus dimensiones, se emprendió apenas unas semanas más tarde. Se había abierto la veda radiactiva. El Balneario de la Aliseda en Jaén publicaba su anuncio de la nueva temporada donde advertía de las bondades del manantial de San José, capaz de desprender en 24 horas 203.6562 litros de aguas de azoe (nitrogenadas) y con grandes cantidades de radiactividad (El Defensor de Córdoba, 3/9/1906), indicadas para "enfermedades del aparato respiratorio, lesiones cardíacas, infartos pulmonares, tuberculosis, tisis, etc." Los anuncios, cerca del millar entre 1906 y 1907, del elegante balneario y hotel que acababa de construir José Salmerón Amat y del que fueron clientes elegantes personalidades de la época como el Infante Carlos de Borbón, padre de María de las Mercedes de Borbón, se extendieron por la geografía, también a través de la prensa regional de la época: El Defensor de Córdoba, El Heraldo Alavés, La defensa (Alcoy), El diario (Orihuela), La Opinión (Santa Cruz de Tenerife), El Castellano (Salamanca) y otros más publicaron sus anuncios. En su mayor parte prensa conservadora o católica, a través de la que este establecimiento, como otros de los que estamos detallando se dirigían a esa burguesía de provincias acomodada que podía asumir los dispendios de una estancia en un balneario.

En los siguientes años, hasta finales de la Primera Guerra Mundial, los balnearios rivalizaron en cualidades terapéuticas de sus aguas y en niveles de radiactividad, que iban desde expresiones genéricas hasta las cifras más exactas, en un alarde de precisión científica.

El agua radiactiva surgía en cada rincón de la geografía española, y con ella las disputas en las páginas de los periódicos. En un anuncio de El Papamoscas (5/7/1908) El Balneario de Alceda en Santander exhibía su radiactividad de 12,40 voltios litro de agua, y apenas

\footnotetext{
${ }^{9}$ En esa fecha no existía una institución con ese nombre. En 1904 se había creado el Laboratorio de Radioactividad por el catedrático de Química José Muñoz del Castillo para conocer los efectos de la radiactividad sobre las aguas minerales, y hasta 1911 no se convirtió en Instituto. Tesis. Radiactividad en España: Ascenso y declive del Instituto de Radiactividad, 1904-1929. Néstor Herrán. Universitat Autònoma de Bacelona. 2006.
} 
unas líneas más abajo en otro anuncio Las Caldas de Oviedo se presentaban como "grandemente radioactivas".

El Hotel balneario de Almeida en Zamora daba un golpe en la mesa en 1909 y en grandes caracteres su publicidad daba cuenta de que su agua llegaba a "10.000 voltios de radiactividad (las que más en el mundo) analizadas por el sabio doctor D. Gabriel de la Puerta" y que entre otras cosas manifestaba que esas "aguas curan la cancerosis". Heraldo de Zamora (29/3/1909).

Los Baños de Zújar en Granada en 1909 prometían 1216 voltios de radiactividad en sus aguas (La Crónica Meridional, 3/5/1909). O les parecía excesiva la cifra de Almeida como para superarla en la publicidad, o simplemente al anunciarse éste en la prensa castellana no llegaba a Andalucía y no eran conscientes de que había un nuevo record con el que no podían rivalizar. Eso sí, a lo largo en sus anuncios, muy habituales en prensa hasta 1915 , presumían que esas aguas eran excelentes remedios para combatir desde el artitrismo hasta la sífilis, y que el balneario estaba construido a imitación del alemán de Wiesbaden y el francés de Air-La- Chapelle, disponía de hoteles y apartamentos familiares. Parafraseando un noticiario por venir, el lujo del mundo entero al alcance de los españoles.

Y apenas unas semanas más tarde esos 1216 voltios eran superados por unas pocas unidades, como en un record de salto de altura. El balneario de la Isabela en Sacedón, Guadalajara, fijaba la radiactividad de sus aguas en los 1227,21 voltios hora litro, "eficacísimas para la curación de todas las enfermedades nerviosas en general, neurastenia, vesania o locura, insomnio, histerismo, epilepsia..." (El Día de Toledo 26/6/1909). Con esa medición en voltios la radiactividad, se hacía una traslación de la aplicación de la electricidad como tratamiento médico. La electricidad se había convertido en esos años en el gran instrumento para publicitar infinidad de artilugios que veían a curar las más diversas dolencias; "la fe en la electricidad era tal que, en la mente de muchos lectores de prensa de fines del siglo XIX, la asociación entre curación y electricidad fue un hecho." (Laguna, 2017: 94). La radiactividad venía a representar la versión modernizada, del siglo XX, de la electricidad decimonónica.

En los siguientes años diversos establecimientos se sumaron a la fiebre radiactiva con más o menos entusiasmo, pero con esa coletilla ineludible para vender unas aguas a la altura de los tiempos, hasta aproximadamente 1917. Uno de los últimos ejemplos lo encontramos en el verano del citado año. Cuando el remozado balneario de Santa Marina en Tarragona publicitaba sus nuevas y magníficas instalaciones y sus aguas purísimas de gran radiactividad (104 voltios por litro) en una campaña que se extendió por los principales diarios de la provincia, desde el Diario de Reus a La Cruz, diario católico o El Restaurador de Tortosa. A partir de entonces, prácticamente nada. La radiactividad desaparecía casi tan abruptamente de las aguas termales, como había llegado. 


\section{La versión urbana de los Balnearios. Los Emanatorios radiactivos}

El negocio del agua radiactiva se amplió más allá de los establecimientos balnearios situados en lugares distantes de las grandes ciudades. Es evidente que acudir a estos centros no estaba al alcance de todas las personas, por disponibilidad, tiempo o dinero. Por esos motivos. el ingenio de diversos avispados empresarios, hoy diríamos emprendedores, o mejor aún promotores de las startup de la época acercaron las portentosas posibilidades del radio a los habitantes de las grandes urbes. Aparecieron primero los balnearios urbanos e inmediatamente su versión sofisticada y modernizada con los Emanatorios Radiactivos.

\section{LOS BALNEARIOS} MAS FAMOSOS

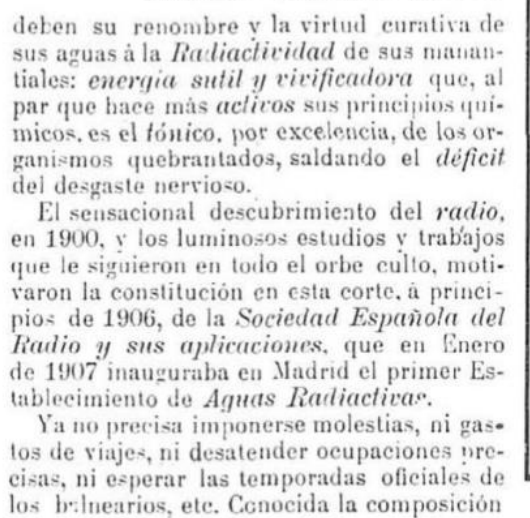
quimica de las más reputadas aguas minera-

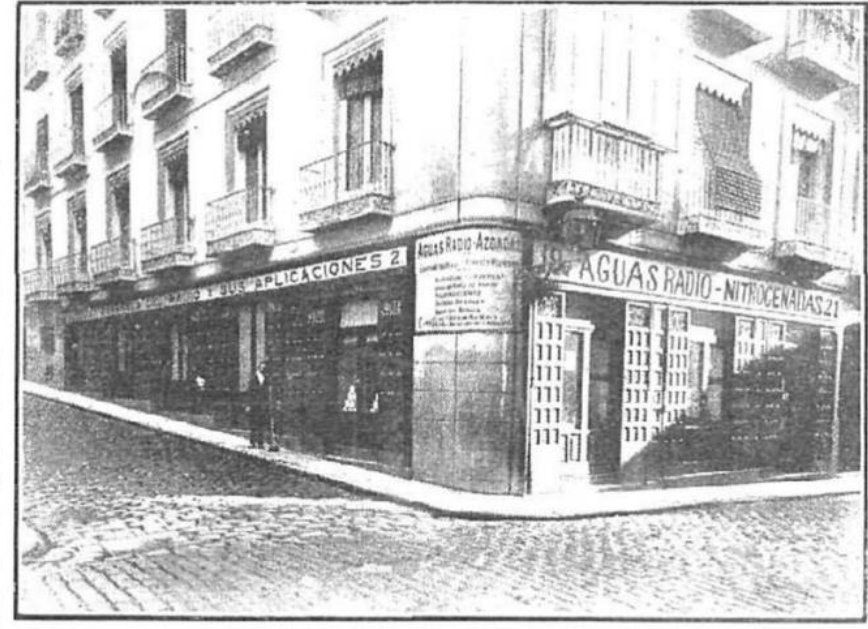

Vista exterior.-IVFANTAS, 19 y 21 .-Te16fone 9.975

les, es empresa facil preparar un baño en el propio domicilio, igual por su composicion, por su temperatura y por su ra. diactividad, sen todo tiempon al alcance de todas las forlunas.

En el citado establecimiento de la calle de las Infantas hay, además, acabadas instalaciones para combatir, ezpeciałmen. te, las enfermedades del aparato respiratorio, con aguas ralionitrogenalas similares a laz de Panticosa. Lirberaaza, CaldaAliseda, etc., y azoe radiuctivo (inhalaciones, pulverizaciones, duchas nasales), con excelente material ad hoc que en la ubltima Exposición de Industrias Madrilenas aicanzó un Premio de Mérito.

VISITESE EL. FST A BL zcimiento: toda suerte de aclaracionez y numerosos testimonios de enfermos y médieos, en los tres años que llera funcionando esta lnstitución.

LNICO EN SU CLASE, no tiene todavia surursales en Madrid ni en provincias.

IMAGEN 3. Publicidad del Balneario urbano de Aguas radiactivas de Madrid rn la revista Nuevo Mundo, 9 noviembre 1909

A partir de mayo de 1909 se inicia una campaña en La Correspondencia de España que anuncia un nuevo establecimiento de baños, creado a inicios de 1907 por la llamada Sociedad Española del Radio, empresa privada que funcionó entre 1903 y 1913, situado en el centro de Madrid en el barrio de Chueca y que venía a ofrecer todos los beneficios de la radioactividad sin las incomodidades de los tradicionales balnearios. La ventaja era además que la terapia podía encargarse en el propio lugar de residencia del paciente. La radiactividad se hacía urbana, popular y a domicilio. Supuestamente el agua se traía de manantiales afamados y una vez en el establecimiento se la irradiaba aún más. 
Los balnearios más famosos deben su renombre y la virtud curativa de sus aguas a la radioactividad de sus manantiales; energía sutil y vivificadora. Ya no precisa imponerse molestias y gastos de viaje, ni desatender ocupaciones precisas, ni esperar las temporadas oficiales de los balnearios, etc. Conocida la composición química de las más reputadas aguas minerales es empresa fácil preparar un baño en el propio domicilio, igual por su composición, por su temperatura, y por su radiactividad, y puesto al alcance de todas las fortunas. (La Correspondencia de España, 30/05/1909).

El siguiente paso fue prescindir del transmisor líquido para la radiactividad y alcanzar un estadio superior de modernización accediendo a la misma a través del aire. Aparecieron los llamados Emanatorios que venían a ser lo más de lo más en radiactividad y métodos de curación. El más destacado sin lugar a dudas fue el llamado Instituto Radiumterápico de Madrid, del cual hemos podido rastrear más de 1500 anuncios insertados en las más importantes cabeceras de la época ${ }^{10}$. Estaba administrado por el médico militar retirado Alfredo Gallego "que se presentaba como especialista en las enfermedades de garganta, nariz, oídos y radiumterapia" (Herrán, 2008: 107).

Situado en el Paseo de Recoletos 31 hasta 1918 y a partir de esa fecha en la no menos importante calle Princesa de la capital de España, el establecimiento publica su primer anuncio el 24 de febrero de 1910 en La Correspondencia de España y El Radical, en el que señalaba que el doctor Gallego había comprado los aparatos de Radium necesarios para curar enfermedades tan diversas como "el cáncer, el lupus, los angiomas, el bocio..." y muchas otras. El mismo anuncio se vanagloriaba: "Apenas se concibe que un solo agente posea fuerza tan grande, misteriosa, verdaderamente maravillosa $y$ constante en sus efectos en tan variadas afecciones".

Fue el inicio de una larga y sostenida campaña en la que junto a algunas primeras aplicaciones del radio en las terapias médicas se deslizaron todo tipo de utilidades en el más claro ejemplo de la publicidad de productos milagro. La inhalación de aire con radiactividad se convertía en un Bálsamo de Fierabrás que todo lo curaba desde el reumatismo a toda clase artritis, neuralgias, afecciones de las vías respiratorias, debilidad senil, gota, inflamaciones del corazón, catarros, raquitismo, enfermedades de la piel y un etc., tan largo como dolencias podamos imaginar. Lo más destacado es que se presentaba como una alternativa superior en efectividad a los populares balnearios, al vencer en las mediciones de lo que hemos venido en llamar la Batalla del voltio. En su publicidad a partir de finales de 1912 afirmaba que el aire durante una sesión en el Instituto era de 43.649 voltios hora por decímetro cúbico, "Lo que demuestra que la

\footnotetext{
${ }^{10}$ Hablamos de prensa de Madrid como La Correspondencia de España, El Imparcial, El Heraldo de Madrid, $A B C$, Blanco y Negro, El Siglo Futuro, El Gedeón y otros, pero también muy variada prensa de provincias como Diario de Córdoba, Heraldo Alavés, Diario de Burgos, La Rioja, El Pueblo (Valencia), El Día de Toledo, Las Provincias (Valencia), y muchos otros más.
} 
proporción de radiactividad en el aire que se respira en este Emanatorio es nueve veces mayor que el que tiene las aguas mineromedicinales más ricas del mundo" (La Correspondencia de España, 10/10,1912).

El siguiente paso del Instituto fue unir la emanación de radium y la electricidad y con ello conseguir lo que denominaban una acción de vigorización total:

Se puede, en esta forma, producir acción sobre la circulación de la linfa y la secreción de las glándulas, la resolución de los exudados, reacción sobre las células, en general, especialmente las nerviosas, aumento de la vitalidad, de la actividad cerebral, de las fuerzas y de la capacidad motriz, mejoría del apetito, regulariza las funciones del estómago é intestinos y disminuye la impotencia y aumenta el volumen de los músculos y su peso. (El Imparcial, 24/2/1914).

Era difícil dar más por menos, porque a continuación se referían todas las dolencias que la combinación de terapias combatía, y que antes hemos referido, añadiendo otras como la diabetes o la parálisis cerebral. Y todo ello avalado por una tecnología que según rezaban los anuncios marchaba al unísono de las principales clínicas de Alemania, Francia e Inglaterra.

La publicidad de Instituto Radiumterápico de Madrid se concentró entre 1910 y 1918, época dorada de la popularidad publicitaria del fenómeno radiactivo, para desaparecer en 1920 donde el descrédito de esas técnicas empezaba a conocerse.

\section{Y para beber, agua radiactiva}

El agua embotellada era concebida en el mercado publicitario de la época como un medicamento en sí misma. Hemos de tener en cuenta en el siglo XIX o principios del siglo XX la gente bebía el agua del pozo o de la fuente, no iban a las tiendas a comprarla, a no ser que adquiriera el estatus de producto casi farmacológico por sus pretendidas propiedades.

Si los balnearios estaban explotando intensamente la pasión por el radium, era bastante previsible que la llamada agua mineromedicinal, la que se concebía como una medicina, y que habitualmente se vendía en las farmacias, proviniendo en gran medida de esos establecimientos termales, se apuntara también a la moda de la radioactividad.

Entre el catálogo de aguas radiactivas destaca por encima de todas la resonancia de las "Aguas Lérez" del balneario de Monte Porreiro. Activado por el indiano Casimiro Gómez que compró unas tierras y manantiales a principios de siglo XX y que en 1904 conseguía la declaración de utilidad pública de su agua al tiempo que edificaba un moderno 
balneario inaugurado en 1906, fue frecuentado por la aristocracia española y buena parte de su clase política. El empresario se lanzó a una exitosa gran campaña de comercialización de sus aguas que incluyeron los mercados de Europa y América ${ }^{11}$, acompañada de un notable plan de marketing para vender las bondades de su mercancía.

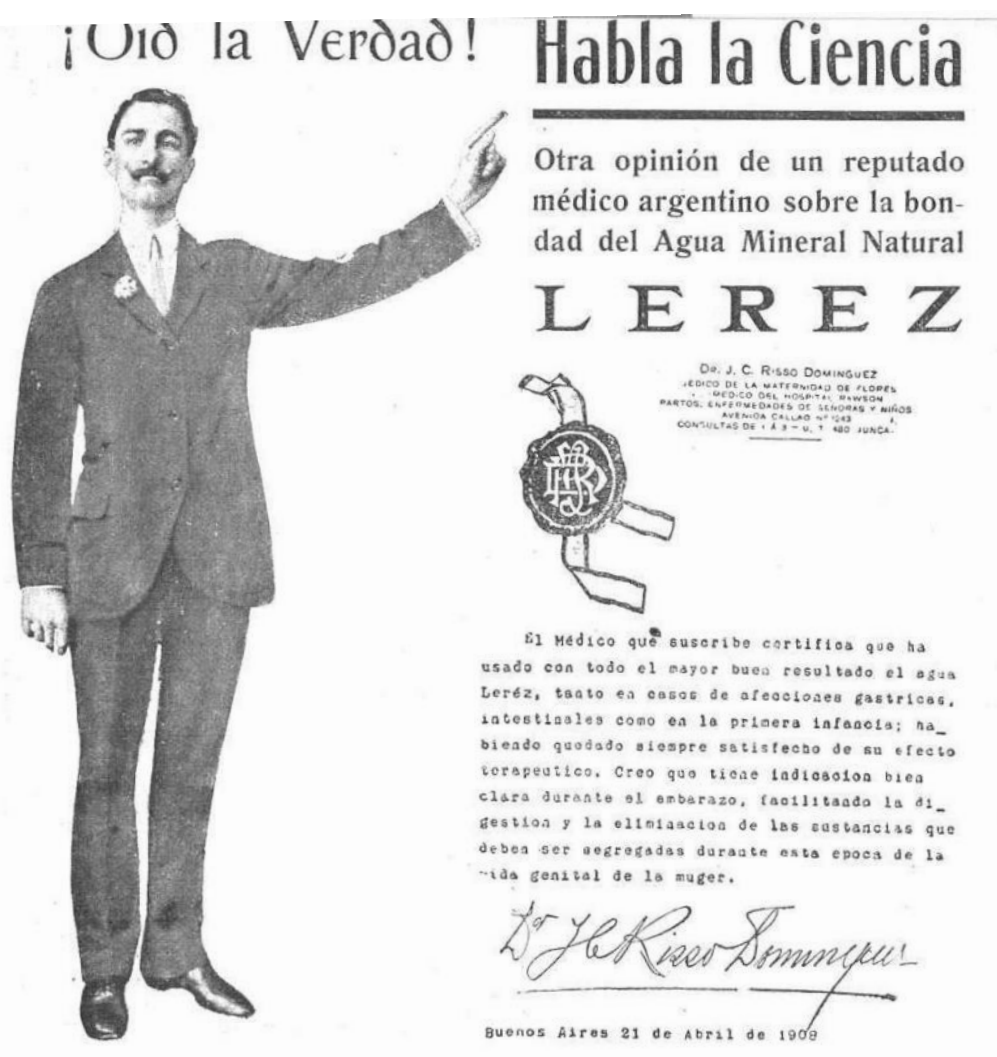

Los maravillosos efectos - terapéuticos de esta agua, se deben no solamente á sus excelentes cualidades medicinales, analizadas y reconocidas por químicos tan reputados como los doctores Miguel Puiggari y Atanasio Qu:roga, sinó aun tanto
ó más á su enorme

EN VENTA EN TODOS LOS BUENOS HOTELES, RESTAURANTS, CONFITERIAS, FARMACIAS Y ALMACENES

\footnotetext{
${ }^{11}$ Se llegó a comentar en la época que llegó a estar presente en la carta del mismísimo Titánic como "Lérez Natural Mineral Water". En Inglaterra creó una Delegación y exportó sus botellas a lugares tan distantes como Estados Unidos, Australia, India o Argentina, donde fue publicitada en la famosa revista Caras y Caretas.
} 
La campaña publicitaria se inicia en 1906, primero en la prensa local como El Faro de Vigo y El Eco de Santiago, coincidiendo con la apertura del nuevo Balneario y el hotel, y a partir de 1907 en la prensa de Madrid: El Heraldo de Madrid, El Liberal, Blanco y Negro, etc. Pero no es hasta 1908 cuando se apunta a la fiebre radiactiva y se pone a competir también en La Batalla del voltio. Según el anuncio (El Liberal, 25/2/1908) las aguas del balneario Villa Buenos Aires, bautizado así por el origen de su fundador, contenían 10.000 voltios por hora y litro; estás, después de su filtrado y embotellado se convertían en las aguas de mesa Lérez que podían comprarse en hoteles, restaurantes, farmacias y droguerías.

Es muy interesante destacar que la popularidad de Lérez y su capacidad de influencia como anunciante se evidencia no tanto en la propia cartelera comercial como en los contenidos periodísticos que protagonizó en las páginas de la prensa. Hablamos de reportajes y noticias diversas, verdaderos publirreportajes encubiertos donde se ilustraba sobe las maravillas del Balneario y sus aguas, en publicaciones como Nuevo Mundo o Heraldo de Madrid a lo largo de 1908 y 1909. Textos donde las fronteras entre información y publicidad quedaban totalmente destruidas. El ejemplo más destacado lo publica Heraldo de Madrid (29/10/1910) en un extenso reportaje a toda página con fotografía sobre un Congreso y Exposición de la Tuberculosis en Barcelona que se aprovechaba para hacer una extensa, intensa y casi podríamos valorar como servil publicidad encubierta de su anunciante. De hecho, el reportaje era una mera excusa para hacer una amplia mención al establecimiento por la existencia de una fuente de la citada agua en la entrada a la Exposición. En el texto de la noticia se deshacían en elogios sobre las propiedades del agua Lérez, y específicamente sobre su elevadísima cifra radiactiva, recordando los 10.000 voltios de su publicidad, avalados por el afamado doctor Muñoz Castillo y toda la batería de enfermedades que era capaz de remediar. El punto culminante de esta habitual confusión entre ciencia y superchería era cuando se ponía en boca del Catedrático de Química y director del Instituto de Radioactividad de Madrid la afirmación: "Cada gota de esta agua-dijo el sabio- es una gota de agua para la salud". No extraña pues que el redactor quisiera dejar claro la irreductible virtud avalada por la ciencia del Agua Lérez: “Quiere, pues, decir la Ciencia con estas afirmaciones que la oportuna mineralización unida a la radiactividad, la hacen de unos efectos curativos superiores a aquellas aguas que aun teniendo una mayor composición química, carecen de esa energía maravillosa y admirable que la Naturaleza reserva en su grado máximo para el agua Lérez".

Ese valor conferido por la autoridad científica sobrevuela constantemente todo este tipo de publicidad que venimos señalando, pero en el caso del agua Lérez fue especialmente insistente y posiblemente eficaz. En la campaña publicitaria que el agua Lérez hizo en Argentina, donde como hemos señalado tuvo una amplia comercialización, podemos observar anuncios a toda página en la que la imagen de un doctor, denominado J.C. 
Risso ${ }^{12}$, del cual figuraba su especialidad y dirección, venía a certificar las propiedades del agua bajo el lema "Oíd la verdad. Habla la Ciencia" en un cuerpo de letra enorme: "Los maravillosos efectos terapéuticos de esta agua, se deben no solamente a sus excelentes cualidades medicinales, analizadas y reconocidas por químicos tan reputados como los doctores Miguel Puiggari y Atanasio Quiropa, sinó aun tanto o más a su enorme RADIACTIVIDAD" (Caras y Caretas, 25/5/1908).

No faltaron otros ejemplos, pero con menos significación y recorrido. A lo largo de 1909 y 1910 El Siglo Futuro, el gran diario carlista publicado entre 1875 y 1936, publicó el anuncio del agua Villa Juana del Manantial San Paulino, catalogada como la mejor agua de mesa y sobre todo la más radiactiva, que se venía en la farmacia de la calle Toledo de Madrid, al respetable previo de 0.65 pesetas la botella. Una cantidad que, de nuevo, nos ilustra sobre el desmesurado precio al que se ofertaban los productos tocados por la magia de lo radiactivo y que por tanto estaban reservados a un público con un elevado poder adquisitivo.

El furor radiactivo llegó a otro tipo de aguas que se habían empezado a popularizar el siglo XIX como fueron las gaseosas en polvo y que en su publicidad tuvieron que hacer frente a las nuevas competidoras. Quizás la más célebre en nuestro país fue el agua del doctor Gustin Lithinés, muy popular durante una buena parte del siglo XX y que en sus anuncios de principio de siglo trataba, después de haber sido símbolo de modernidad, de resistir el embate de la moda: "No descompone el vino, se mezcla a todas bebidas y es muy superior á muchas Aguas más o menos naturales, las cuales embotelladas pierden toda su radioactividad. No resultan sino ser aguas muertas" (La Correspondencia de Valencia, 29/12/1916).

12 El mencionado doctor apareció en la misma revista certificando las bondades de otros medicamentos o productos, como la Leche infantil Horlicks. 


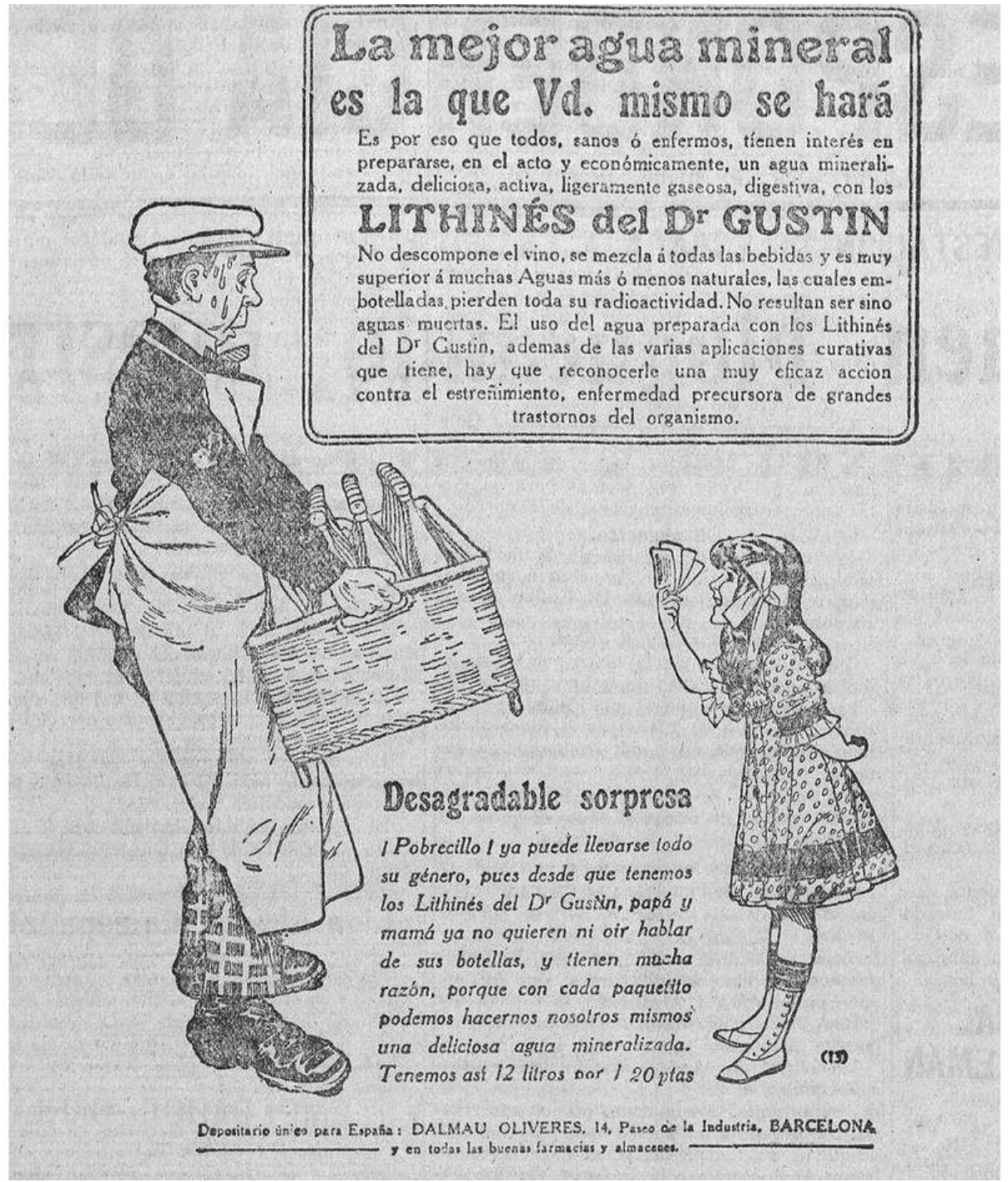

IMAGEN 5. Anuncio del agua carbonatada del Doctor Gustín Lithinés en La Correspondencia de España, 29 diciembre 1916

En todo caso, a esas alturas el entusiasmo por el radio estaba empezando a remitir. A partir de 1918 empieza a escasear la presencia del término radiactivo de la cartelera de la prensa española, y a partir de los años 20 la publicidad radiactiva es muy escasa y puntual, la fiebre ha remitido, aunque volverá algún pico puntual. Las investigaciones avanzaban y empezaban a advertir de las consecuencias negativas de una exposición a tales sustancias. La presencia del radium en la cartelera comercial se limitará desde esos momentos a los anuncios de los Gabinetes Médicos que en sus servicios de exploración lo hacen aparecer como método de diagnóstico junto con los Rayos $\mathrm{X}$, signos de modernidad bajo control. 


\section{Referencias bibliográficas:}

ALMUDÉVER-CAMPO, L. y CAMAÑO-PUIG, R. (2007): “Publicidad y cáncer en la prensa escrita” en Ámbitos, Revista Internacional de Comunicación, no 47, pp. 195-211.

CAMPANARIO, J.M, MOYA, A. y OTERO, J. (2001): "Invocaciones y usos ineducados de la ciencia en la publicidad" en Enseñanza de la Ciencias, 2001, 19(1), 45-56.

DRAGONI, S.A. (2012): Héroes y villanos de la medicina. Las dos caras de la misma moneda, Buenos Aires, Editorial Dunken.

HERRÁN, N. (2008): Aguas, semillas y radiaciones: El laboratorio de radiactividad de la Universidad de Madrid, 1904-1929 (Estudios sobre la ciencia), Madrid, CSIC.

LAGUNA, A. (2018): Salud, sexo y publicidad. Los inicios de la publicidad de masas, Cuenca, Ediciones de la Comunidad de Castilla La Mancha.

MOORE, K. (2018): Las chicas del Radio, Madrid, Capitán Swing.

VALLEJO-POUSADA, R. (2015) "Salud y recreo: los balnearios de Galicia y el descubrimiento de una periferia turística en el primer tercio del siglo XX" en Agua y territorio, Núm. 6, pp. 62-79, JAÉN, Universidad de Jaén.

VILAR, M y LINDOSO, E. “La explotación empresarial de las aguas mineromedicinales: la industria del agua embotellada en España 8c. 1850-2012)". XI Congreso Internacional de la Asociación de Historia Económica. 4-5 septiembre 2014. Madrid. Disponible en Internet el 17 de diciembre 2019.

https://www.labrujulaverde.com/2017/02/el-caso-radithor-y-la-moda-de-losmedicamentos-radiactivos-de-los-anos-veinte. Disponible en Internet el 13 de enero 2020.

https://www.investigacionyciencia.es/blogs/fisica-y-quimica/24/posts/aguasminerales-radiactivas-11822. Disponible en Internet el 20 de enero 2020.

https://pontevedraviva.com/opinion/2500/balneario-monte-porreiro-jose-benito-g 